Marquette University

e-Publications@Marquette

Physics Faculty Research and Publications

Physics, Department of

$1-1-2009$

Field Induced Domain Wall Collisions in Thin Magnetic Nanowires

Andrew Kunz

Marquette University, andrew.kunz@marquette.edu

Published version. Applied Physics Letters, Vol. 94, No. 13 (2009). DOI. (C) 2009 American Institute of Physics. Used with permission. 


\title{
Field induced domain wall collisions in thin magnetic nanowires
}

\author{
Andrew Kunz ${ }^{\mathrm{a})}$ \\ Department of Physics, Marquette University, Milwaukee, Wisconsin 53233, USA
}

(Received 26 January 2009; accepted 12 March 2009; published online 31 March 2009)

\begin{abstract}
In a two-dimensional magnetic nanowire, it is possible to engineer collisions between two domain walls put into motion by an externally applied field. We show that the topological defects that define the domain wall can be controlled to allow for both domain wall annihilation and preservation during the collisions as long as the wire remains thin. The preservation process can be used to release pinned domain walls from notches with small applied fields. () 2009 American Institute of Physics. [DOI: 10.1063/1.3112577]
\end{abstract}

Understanding the dynamic properties of a moving magnetic domain wall in a thin, narrow nanowire is a challenge for fundamental physics and for technological applications in magnetic recording and sensing. In a thin, narrow wire the magnetic moments of the material lie in the plane of the wire, oriented along the long axis due to the strong shape anisotropy. ${ }^{1-3}$ Although energetically unfavorable, a domain wall may exist in the wire separating regions of opposite magnetization. The simplest domain wall is a transverse wall with head to head or tail to tail alignment with the magnetic moments rotating through a total of $180^{\circ}$. Fast, reliable, and controllable motion of the domain wall is essential for the operation of several proposed nanowire storage, logic, and sensing devices. ${ }^{4,5}$ There is a large effort to understand and control the motion of a single domain wall with lithographic patterning techniques, current pulses, and tailored fields; ${ }^{6-12}$ however it will be necessary to inject and move several domain walls concurrently for practical application. ${ }^{5,13}$

Electric currents and externally applied magnetic fields are the two principle mechanisms for driving a domain wall along a wire. A current is known to each domain wall in the same direction, maintaining the overall magnetic structure in the wire. ${ }^{14}$ However, when a magnetic field is used to move two domain walls, the domain walls move in opposite directions and collide. In this letter it is shown that the collision is capable of preserving the domain configuration when the structure of the colliding domain walls is properly controlled.

A magnetic domain wall can be considered a composite object consisting of two or more topologic defects. In a magnetic nanowire, the possible defects are vortices and antivortices with winding number of $n= \pm 1$, and edge defects with half-integer winding number $n= \pm \frac{1}{2} .15$ Possible winding numbers and their relevant magnetic structure is represented in Fig. 1(a). The simplest domain wall in a thin nanowire consists of two elementary edge defects of opposite winding number for a net composite charge of zero. ${ }^{15}$ Two possible composite structures are presented in Fig. 1(b). It is known that the annihilation requires an interaction between defects with opposite topological charge. ${ }^{16}$ Therefore, by suitable creation of the domain structure, both domain wall annihilation and preservation can be realized.

The tool used for visualization of the collisions in a magnetic nanowire is a micromagnetic simulation utilizing

${ }^{a)}$ Electronic mail: andrew.kunz@marquette.edu. the Landau-Lifshitz equation of motion for a magnetic moment $m$

$$
\frac{\partial \vec{m}}{\partial t}=-\gamma(\vec{m} \times \vec{H})-\frac{\alpha \gamma}{M_{s}} \vec{m} \times(\vec{m} \times \vec{H}),
$$

where $\gamma$ is the gyromagnetic ratio, $\alpha$ is the phenomenological damping parameter, $M_{s}$ is the saturation magnetization, and $H$ is the total field experienced by the moment. ${ }^{17} \mathrm{~A}$ fourth order predictor-corrector integration time step of less than a picosecond and phenomenological damping of $\alpha$ $=0.008$ are used to complete the simulations. The materials parameters are those typical of Permalloy with saturation magnetization $M_{s}=8.0 \times 10^{5} \mathrm{~A} / \mathrm{m}$, exchange stiffness $A$ $=1.3 \times 10^{-11} \mathrm{~J} / \mathrm{m}$, and no crystalline anisotropy. Wires with rectangular cross sections width ranging from 50 to $300 \mathrm{~nm}$ and thickness ranging from 2 to $10 \mathrm{~nm}$ were simulated. The results presented below are dependent on the thickness. A field-driven domain wall moves quickest, and with the most consistent structure, for fields less than the critical Walker field where vortices and antivortices nucleate in the domain wall. ${ }^{9,18}$ For this reason, the driving fields used in the simulations are all under $15 \mathrm{Oe}$, which allows for easy control of the magnetic structure.

Two $180^{\circ}$ domain walls are inserted at different locations along the length of the wire, creating three longitudinal domains. As shown in time-lapse sequence of Figs. 2(a)-2(d), the magnetic moments in each domain wall are aligned parallel to each other along the $+y$-axis and separate a reversed magnetic domain in the center of the wire $(-x$-axis) from the two end domains ( $+x$-axis). Experimentally a domain wall can be injected into a wire with the use

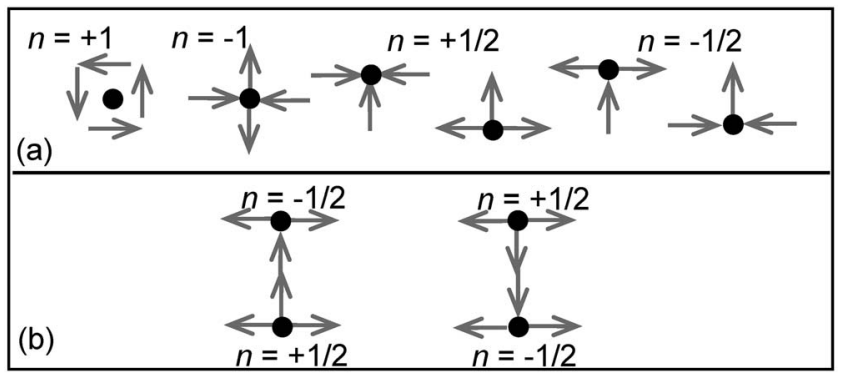

FIG. 1. (a) Schematic representations of a magnetic vortex (winding number $n=1)$, antivortex $(n=-1)$, and their half-integer equivalents. A domain wall has a total winding number of zero and in (b) is shown to be composed of two half-vortices of opposite winding number. 


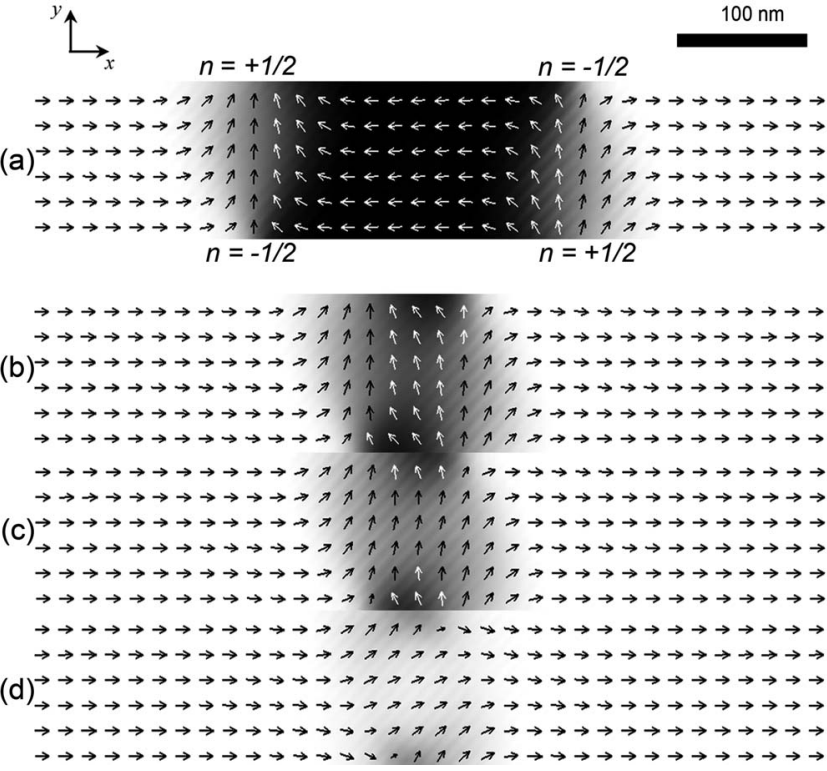

FIG. 2. Time-lapse sequence of two domain walls being driven by a 10 Oe field aligned along the $+x$-axis in a $5 \mathrm{~nm}$ thick wire. In (a) the elementary topologic charges are given. Opposite topologic charges annihilate.

of an injection pad attached to one end of the wire. ${ }^{11}$ The magnetic structure of the wall can be controlled by the use of a bias field which can change the magnetic state of the pad leaving the wire unaffected. The elementary topological charges for each wall are given in Fig. 2(a). When a 10 Oe external field is applied along the $+x$-axis the domains oriented along the field direction grow at the expense of the reverse domain as expected. When the domain walls meet, each edge defect annihilates with an edge defect of opposite topological charge. The annihilation takes place through a reversal of the center reversed domain into the direction of the domain wall magnetization and energy is dissipated through the production of spin waves in the wire.

The simulation is repeated exactly as above, but with the magnetization (and therefore the winding numbers) of the right-hand domain wall reversed as shown in the time-lapse sequence in Figs. 3(a)-3(d). The applied field drives the domain walls toward each other, but in this case the walls do not annihilate when they collide. To annihilate, two defects of opposite topological charge must interact or a pair of charges must be created ${ }^{19-22}$ and in this case at each edge the interacting topologic charges are the same. The time lapse shows that prior to the collision the central domain shrinks but instead of rotating into the direction of the domain walls as before, the rotation process is frustrated. The best path to reversal is to rotate out-of-plane but the strong shape anisotropy of the thin wire inhibits this process. The walls bounce off of each other before relaxing into the final equilibrium structure shown in Fig. 3(d). The final structure is a $360^{\circ}$ domain wall. ${ }^{23-29}$ Wires ranging in cross-section width from 50 to $300 \mathrm{~nm}$ wire simulated and in each case when the thickness was increased beyond $8 \mathrm{~nm}$ the domain walls annihilated upon contact. Keeping the wire thin, and the driving fields below the critical Walker field, helps the system maintain a two-dimensional magnetic structure. As the thickness of the wire grows, the full skyrmion charge, which is a product of the winding number and the out-of-plane magnetization (polarization) of a topologic defect, becomes important. ${ }^{16,19}$ Due to the first term of the LL Eq. (1), each
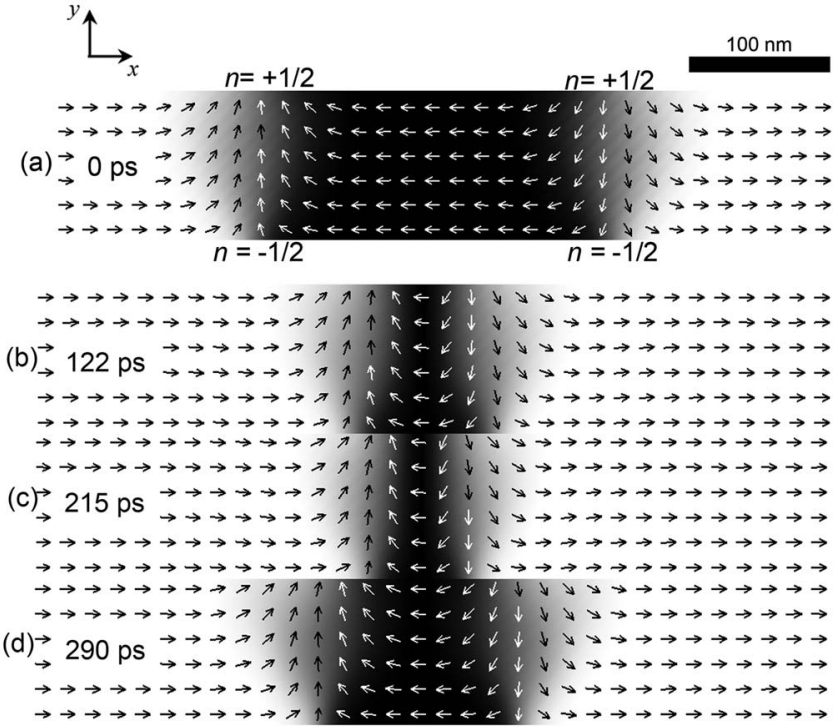

FIG. 3. Time-lapse sequence of two domain walls being driven by a 10 Oe field aligned along the $+x$-axis in a $5 \mathrm{~nm}$ thick wire. In (a) the elementary topologic charges are given. The topologic charges are preserved leading to the $360^{\circ}$ domain wall (d).

domain wall rotates about the applied field (in a clockwise direction when looking along the direction of the applied field) giving rise to an opposite skyrmion charge at each of the interacting edge defects and leading to a dynamic path of annihilation. However, in the thin $100 \times 5 \mathrm{~nm}^{2}$ cross-section wire the domain structure is robust so that a field of over 600 Oe is necessary to remove the $360^{\circ}$ wall. At the same time the domain walls are weakly coupled such that a 30 Oe field can separate them back into two individual $180^{\circ}$ walls.

The collision process can be used to release a domain wall that is pinned by a notch as shown in the time-lapse series of Figs. 4(a)-4(d). Domain wall pinning is critical for many proposed devices but removing the wall from the notch requires large fields, current pulses, or laser heating. In this case a domain wall is held in place by a small notch which requires a minimum field of 80 Oe to release it. The left wall is driven along the wire by a 10 Oe field until it collides with the pinned wall. The pinned wall is forced out of the notch

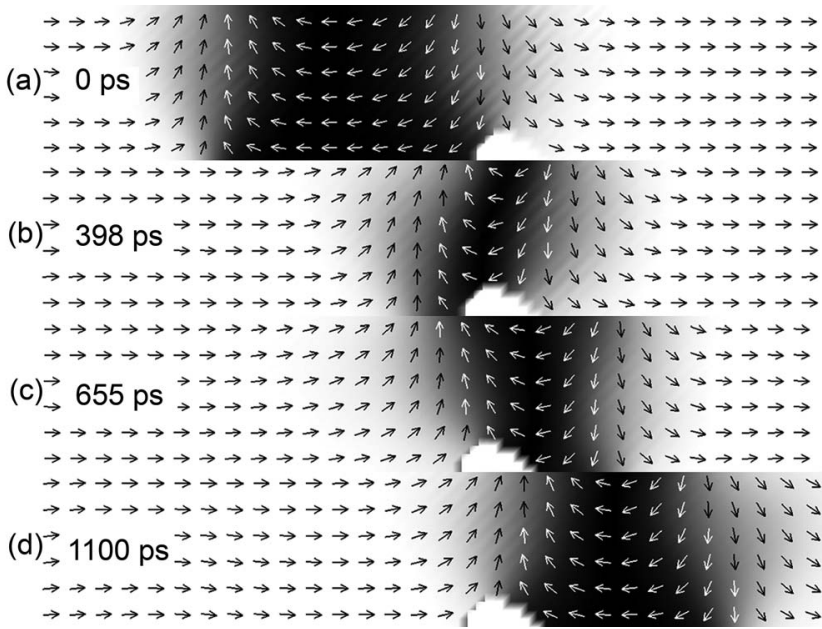

FIG. 4. Time-lapse sequence of a collision between a wall driven by a $10 \mathrm{Oe}$ field and a wall pinned by a small notch. The collision drives the pinned wall from the notch, which captures the moving wall. 
during the collision. This wall will then be shifted down the wire by reversing the applied field.

In summary, by preparing the domain walls with the correct magnetization, it is possible to preserve the walls in a field-driven collision. The elementary winding numbers for each of the colliding domain walls must be identical, and the nanowire must behave two-dimensionally to hold the magnetic moments in the plane of the wire. As the wire thickness is increased, the magnetization is capable of rotating out of the plane and the walls annihilate. The collisions can be used to release pinned domain walls with weak external fields.

This work was financially supported by a Cottrell College Science Award from Research Corporation and by the National Science Foundation (Grant No. DMR-0706194). The author also acknowledges the support of the Helen Way Klingler College of Arts and Sciences at Marquette University.

${ }^{1}$ J.-Y. Lee, K.-S. Lee, S. Choi, K. Y. Guslienko, and S.-K. Kim, Phys. Rev. B 76, 184408 (2007).

${ }^{2}$ R. D. McMichael and M. J. Donahue, IEEE Trans. Magn. 33, 4167 (1997).

${ }^{3}$ O. A. Tretiakov, D. Clarke, G.-W. Chern, Y. B. Bazaliy, and O. Tchernyshyov, Phys. Rev. Lett. 100, 127204 (2008).

${ }^{4}$ D. A. Allwood, G. Xiong, C. C. Faulkner, D. Atkinson, D. Petit, and R. P. Cowburn, Science 309, 1688 (2005).

${ }^{5}$ S. S. P. Parkin, M. Hayashi, and L. Thomas, Science 320, 190 (2008).

${ }^{6}$ D. Petit, A.-V. Jausovec, H. T. Zeng, E. Lewis, L. O’Brien, D. Read, and R. P. Cowburn, Appl. Phys. Lett. 93, 163108 (2008).

${ }^{7}$ A. Vanhaverbeke, A. Bischof, and R. Allenspach, Phys. Rev. Lett. 101, 107202 (2008).

${ }^{8}$ J.-Y. Lee, K.-S. Lee, and S.-K. Kim, Appl. Phys. Lett. 91, 122513 (2007).
${ }^{9}$ A. Kunz and S. C. Reiff, Appl. Phys. Lett. 93, 082503 (2008).

${ }^{10}$ A. Kunz and S. C. Reiff, J. Appl. Phys. 103, 07 D903 (2008).

${ }^{11}$ S. Glathe, R. Mattheis, and D. V. Berkov, Appl. Phys. Lett. 93, 072508 (2008).

${ }^{12}$ L. Thomas, M. Hayashi, X. Jiang, R. Moriy, C. Rettner, and S. S. P. Parkin, Nature (London) 443, 197 (2006).

${ }^{13}$ M. Hayashi, L. Thomas, R. Moriya, C. Rettner, and S. S. P. Parkin, Science 320, 209 (2008).

${ }^{14}$ A. Yamaguchi, T. Ono, S. Nasu, K. Miyake, K. Mibu, and T. Shinjo, Phys. Rev. Lett. 92, 077205 (2004)

${ }^{15}$ O. Tchernyshyov and G.-W. Chern, Phys. Rev. Lett. 95, 197204 (2005).

${ }^{16}$ O. A. Tretiakov and O. Tchernyshyov, Phys. Rev. B 75, 012408 (2007)

${ }^{17}$ LLG MICROMAGNETIC SIMULATOR (http:llgmicro.home.mindspring.com/) v.2.61 (2007)

${ }^{18}$ N. L. Schryer and L. R. Walker, J. Appl. Phys. 45, 5406 (1974).

${ }^{19}$ R. Hertel and C. M. Schneider, Phys. Rev. Lett. 97, 177202 (2006).

${ }^{20}$ K. Y. Guslienko, K.-S. Lee, and S.-K. Kim, Phys. Rev. Lett. 100, 027203 (2008).

${ }^{21}$ K.-S. Lee, K. Y. Guslienko, J.-Y. Lee, and S.-K. Kim, Phys. Rev. B 76, 174410 (2007).

${ }^{22}$ R. Hertel, S. Gilga, M. Fanhle, and C. M. Schneider, Phys. Rev. Lett. 98, 117201 (2007).

${ }^{23}$ X. Portier and A. K. Petford-Long, Appl. Phys. Lett. 76, 754 (2000).

${ }^{24}$ J. Rothman, M. Klaui, L. Lopez-Diaz, C. A. F. Vaz, A. Bleloch, J. A. C. Bland, Z. Cui, and R. Speaks, Phys. Rev. Lett. 86, 1098 (2001).

${ }^{25}$ M. Klaui, C. A. F. Vaz, J. A. C. Bland, L. J. Heyderman, F. Nolting, A. Pavlovska, E. Bauer, S. Cherifi, S. Heun, and A. Locatelli, Appl. Phys. Lett. 85, 5637 (2004).

${ }^{26}$ F. J. Castano, C. A. Ross, C. Frandsen, A. Eilez, D. Gil, H. I. Smith, M. Redjdal, and F. B. Humphrey, Phys. Rev. B 67, 184425 (2003).

${ }^{27}$ G. D. Chaves-O'Flynn, K. Xiao, D. L. Stein, and A.D. Kent, J. Appl. Phys. 103, 07D917 (2008).

${ }^{28}$ C. B. Muratov and V. V. Osipov, J. Appl. Phys. 104, 053908 (2008).

${ }^{29}$ J.-G. Zhu and Y. Zheng, in Spin Dynamics in Confined Magnetic Structures I, edited by B. Hillebrands and K. Ounadjela (Springer, Berlin, Heidelberg, 2002). 